\title{
BORDER-COLLISION BIFURCATIONS IN ONE-DIMENSIONAL DISCONTINUOUS MAPS
}

\author{
PARAG JAIN \\ Department of Mechanical Engineering, \\ Stanford University, CA 94305, USA \\ parag_jain@lycos.com \\ SOUMITRO BANERJEE \\ Department of Electrical Engineering, \\ Indian Institute of Technology, Kharagpur 721302, India \\ soumitro@ee.iitkgp.ernet.in
}

Received June 27, 2002; Revised September 6, 2002

\begin{abstract}
We present a classification of border-collision bifurcations in one-dimensional discontinuous maps depending on the parameters of the piecewise linear approximation in the neighborhood of the point of discontinuity. For each range of parameter values we derive the condition of existence and stability of various periodic orbits and of chaos. This knowledge will help in understanding the bifurcation phenomena in a large number of practical systems which can be modeled by discontinuous maps in discrete domain.
\end{abstract}

Keywords: Border-collision bifurcations; discontinuous maps; power electronics.

\section{Introduction}

In recent years, the theory of border-collision bifurcations has been developed for piecewise smooth maps [di Bernardo et al., 1999; Banerjee et al., 2000a; Banerjee et al., 2000b] of the form

$$
f(x, y, \rho)= \begin{cases}f_{1}(x, y, \rho) & \text { for }(x, y) \in R_{1} \\ f_{2}(x, y, \rho) & \text { for }(x, y) \in R_{2} \\ \vdots & \\ f_{n}(x, y, \rho) & \text { for }(x, y) \in R_{n}\end{cases}
$$

where $\rho$ is a parameter and $R_{1}, R_{2}$, etc. are different regions of the phase space, with borderlines dividing these regions.

These works assume that the map is continuous, but with a discontinuity in the Jacobian across the borderlines. Though investigation along this line was initiated as a mathematical possibility [Nusse \& Yorke, 1992, 1995], the recent spurt of activity was prompted by the discovery that a large class of practical systems in electrical engineering can be modeled as piecewise smooth maps, and border-collision bifurcations are quite common in them [Yuan et al., 1998; Banerjee et al., 1997] (a review is available in [Banerjee \& Verghese, 2001]). Though in general the Jacobian elements were assumed to be finite, investigations on the dynamics of the impact oscillator [Budd, 1995; Budd \& Dux, 1994; Nusse et al., 1994; Chin et al., 1995] considered maps with square-root singularity at the border.

Another line of development has shown that there are important classes of switching systems - like thyristor circuits [Dobson, 1995; Rajaraman et al., 1996; Jalali et al., 1996] and the Colpitts oscillator [Maggio et al., 2000] — that yield discontinuous maps under discrete-time modeling, and nonstandard bifurcation phenomena can occur in such systems. However the theory for understanding such bifurcation phenomena is not available yet. In this 
paper we present the first attempt to classify the border-collision bifurcations that occur in onedimensional maps with discontinuity at the border. We also illustrate the application of the theory in a practical example of a power electronic switching circuit with a delay in feedback loop.

\section{The One-Dimensional Discontinuous Map}

Consider a 1-D map $f(x ; \mu)$ that maps the real line $\mathbb{R}^{1}$ to itself and depends smoothly on the parameter $\mu$. A point $x=x_{b}(\mu)$ on the real line divides it into two regions $R_{A}$ and $R_{B}$. The map $f(x ; \mu)$ is piecewise continuous in the sense that it is continuous in $(x ; \mu)$ on each of the regions $R_{A}$ and $R_{B}$, but is discontinuous at $x_{b}$. In particular, the one-sided limits of the partial derivatives of $f(x ; \mu)$ must exist at the border $x_{b}$. Without loss of generality, we consider maps whose partial derivatives at the border and the length of the discontinuity at the border are independent of the parameter $\mu$.

Since we are interested only in the bifurcations that occur when a fixed point crosses the point of discontinuity $x_{b}$, we can study these through the piecewise linear approximation in the neighborhood of the border:

$$
x_{n+1}= \begin{cases}a x_{n}+\mu & \text { for } x_{n}<0 \\ b x_{n}+\mu+l & \text { for } x_{n}>0\end{cases}
$$

where the state space is divided into two halves $\mathrm{L}$ (left) and $\mathrm{R}$ (right), and $l$ is the length of the discontinuity. A transformation of coordinates moved the break-point to the origin. The fixed point in $\mathrm{L}$ is located at $x_{L}^{*}=\mu /(1-a)$ and that in $\mathrm{R}$ is located at $x_{R}^{*}=(\mu+l) /(1-b)$. The left half of the map intersects the $45^{\circ}$ line for $\mu<0$ and the right half intersects this line at $\mu>-l$. This means that the fixed point $x_{L}^{*}$ collides with the border at $\mu=0$ and the fixed point $x_{R}^{*}$ collides with the border at $\mu=-l$. Therefore we can expect two border-collision events as the parameter $\mu$ is varied.

\section{The Classification}

\subsection{Case 1: $0<a<1$ and $0<b<1$}

We first consider the case of positive $l$, as illustrated in Fig. 1. In this case there is a stable fixed point for $\mu<-l$ and for $\mu>0$, and there are two stable fixed points $x_{L}^{*}$ and $x_{R}^{*}$ at the two sides of the discontinuity for $-l<\mu<0$. Multiple fixed points can occur in continuous maps also, but there is an important difference. In a continuous map, there must be an unstable fixed point between the stable fixed points while there is no such requirement in a discontinuous map. For the continuous map, the unstable fixed point acts as the boundary between the two basins of attraction, while in the discontinuous map the point of discontinuity separates the two basins. The distinctive feature of a discontinuous map (see Fig. 1) is that single fixed points may appear or disappear, while in continuous maps they can appear or disappear only in pairs.

The bifurcation behavior is entirely different if the discontinuity $l$ is negative. There is a stable fixed point each for $\mu<0$ and for $\mu>-l$, and there is no fixed point for $-l>\mu>0$. For $-l>\mu>0$, orbits in the left half move towards the right and those is the right half move towards the left, enabling stable high-periodic orbits to exist.

Since the map is linear in each half, high-period orbits cannot exist with all points in $\mathrm{L}$ or all points in $\mathrm{R}$. The period-2 fixed point must be of LR type, with the point in L given by $(b \mu+\mu+l) /(1-a b)$ and the point in $\mathrm{R}$ given by $(a \mu+\mu+a l) /(1-a b)$.
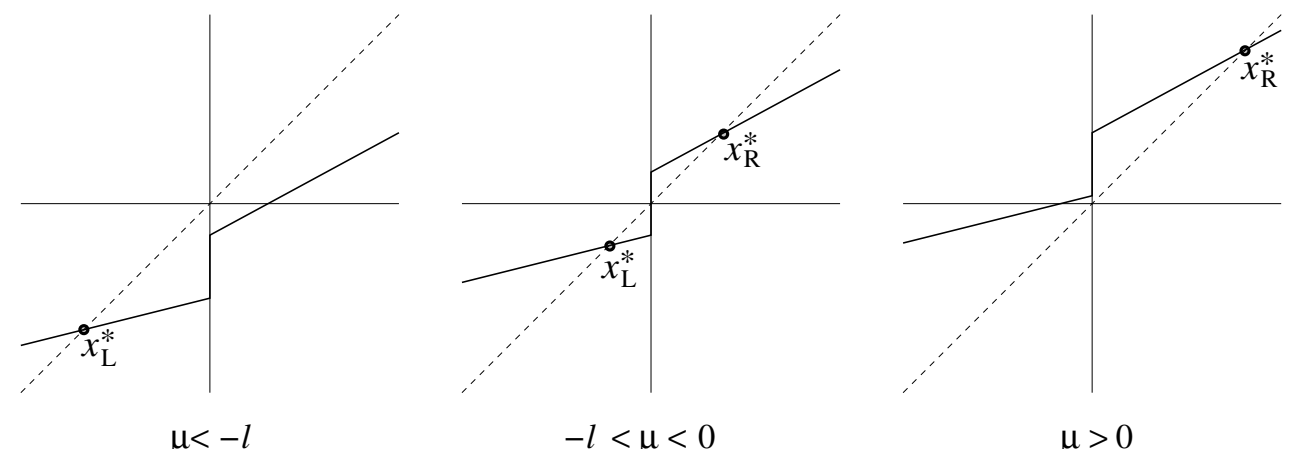

Fig. 1. Graphs of the map for $0<a<1$ and $0<b<1$, and $l>0$. 
This orbit will exist so long as

$$
\frac{b \mu+\mu+l}{1-a b}<0 \text { and } \quad \frac{a \mu+\mu+a l}{1-a b}>0 .
$$

There can be two types of period-3 orbits - LRR type and LLR type. For the LRR type, the three points in the orbit are $\left(b^{2} \mu+b \mu+b l+\mu+l\right) /\left(1-a b^{2}\right)$, $(a b \mu+a b l+a \mu+a l+\mu) /\left(1-a b^{2}\right)$, and $(a b \mu+a b l+$ $b \mu+\mu+l) /\left(1-a b^{2}\right)$. This orbit will exist so long as

$$
\begin{gathered}
\frac{b^{2} \mu+b \mu+b l+\mu+l}{1-a b^{2}}<0, \\
\frac{a b \mu+a b l+b \mu+\mu+l}{1-a b^{2}}>0
\end{gathered}
$$

Similarly, the three points in an LLR orbit are $(a b \mu+b \mu+\mu+l) /\left(1-a^{2} b\right),(a b \mu+a \mu+a l+m) /(1-$ $\left.a^{2} b\right)$, and $\left(a^{2} \mu+a^{2} l+a \mu+\mu\right) /\left(1-a^{2} b\right)$. This orbit will exist in the parameter range given by

$$
\frac{a b \mu+a \mu+a l+m}{1-a^{2} b}<0, \quad \frac{a^{2} \mu+a^{2} l+a \mu+\mu}{1-a^{2} b}>0
$$

The range of existence of the higher iterates can also be obtained in a similar manner, but the expressions become cumbersome and we exclude them here.

From the above, a few conclusions can be drawn. First, since $a$ and $b$ are both less than unity, if any high-periodic orbit exists it is stable, and there can be no chaotic orbit since the map is not stretching. Second, there will be one range of the parameter where period-2 orbit will exist, two ranges of the parameter where period-3 orbit will exist, and similarly the period- $n$ orbit will exist in $(n-1)$ ranges of the parameter. Third, the

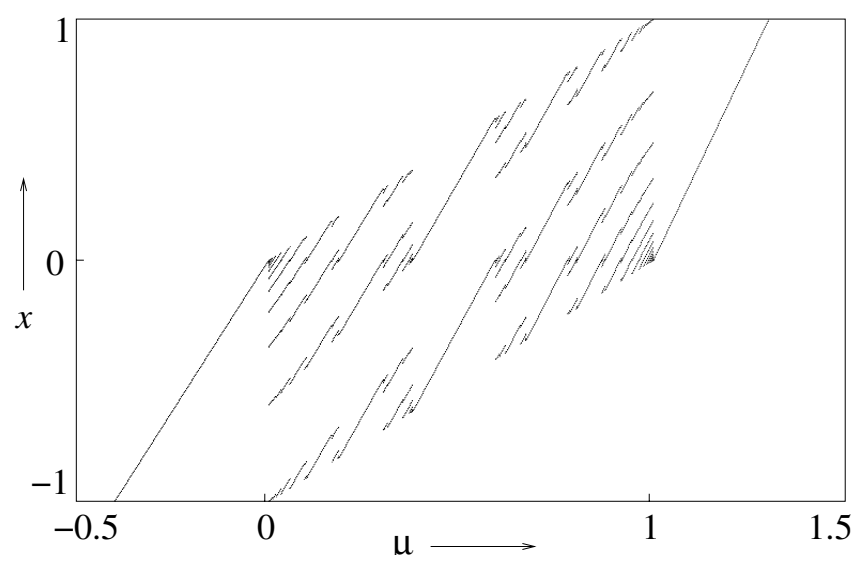

Fig. 2. Bifurcation diagram for the case $0<a<1,0<b<$ 1 , and $l<0$. ranges of occurrence of orbits of consecutive periodicity (e.g. period-2 and period-3) are not continuous for $0<a<1$ and $0<b<1$, and hence the order of occurrence of high-periodic orbits in the range $-l>\mu>0$ will be nonmonotonic. The above phenomena are observed in the bifurcation diagram (Fig. 2).

\subsection{Case 2: $0<a<1$ and $b>1$}

If the discontinuity is positive, for $\mu<-l$ a stable fixed point exists in $\mathrm{L}$, with basin of attraction $\left(-\infty, x_{R}^{*}\right)$. Initial conditions to the right of fixed point $x_{R}^{*}$ go to infinity. For $-l<\mu<0$, the fixed point $x_{L}^{*}$ remains stable but the basin boundary moves to the point of discontinuity. And at the critical value of $\mu=0$, the fixed point disappears and all trajectories go to infinity. For practical systems such a situation may prove disastrous since the stability margin (or the slope) of the map at the fixed point gives no indication of the impending catastrophe.

A reverse bifurcation behavior is observed for $a>1$ and $0<b<1$ where no fixed point exists and all trajectories go to $-\infty$ for $\mu<-l$. For $-l<\mu<0, x_{R}^{*}$ exists and is stable with a basin of attraction $(0, \infty)$. For $\mu>0, x_{L}^{*}$ also exists but is unstable and so the basin boundary shifts to $x_{L}^{*}$. All other initial conditions go to $-\infty$.

If the discontinuity is negative, for $\mu<0$ two fixed points exist $-x_{L}^{*}$ stable and $x_{R}^{*}$ unstable resulting in a period- 1 orbit with basin of attraction $\left(-\infty, x_{R}^{*}\right)$. As $\mu$ exceeds zero, the fixed point $x_{L}^{*}$ disappears while $x_{R}^{*}$ remains unstable. But since orbits in $x<0$ move to the right and orbits in $x>0$ move to the left, high-periodic orbits can exist so long as $\mu<-l / b$. As $\mu$ is varied in $0<\mu<-l$, the periodicity varies nonmonotonically. But since $b>1$, eventually the orbit becomes globally stretching and hence chaotic at some parameter value. The basin of this chaotic attractor remains $\left(-\infty, x_{R}^{*}\right)$. All initial conditions outside this basin go monotonically to $+\infty$. This chaotic attractor becomes unstable for $\mu>-l / b$ when all trajectories go monotonically to $+\infty$. For $\mu>-l$, the fixed point $x_{R}^{*}$ disappears and there can be no stable orbit. The resulting bifurcation diagram is shown in Fig. 3.

An opposite situation is observed for $a>1$ and $0<b<1$ where a chaotic attractor becomes stable for $\mu>-(l(a-1) / a)$ prior to which all trajectories go monotonically to $-\infty$. The basin of this chaotic attractor is $\left(x_{L}^{*}, \infty\right)$. As $\mu$ is increased, highperiodic orbits become stable, and finally for $\mu>-l$ 


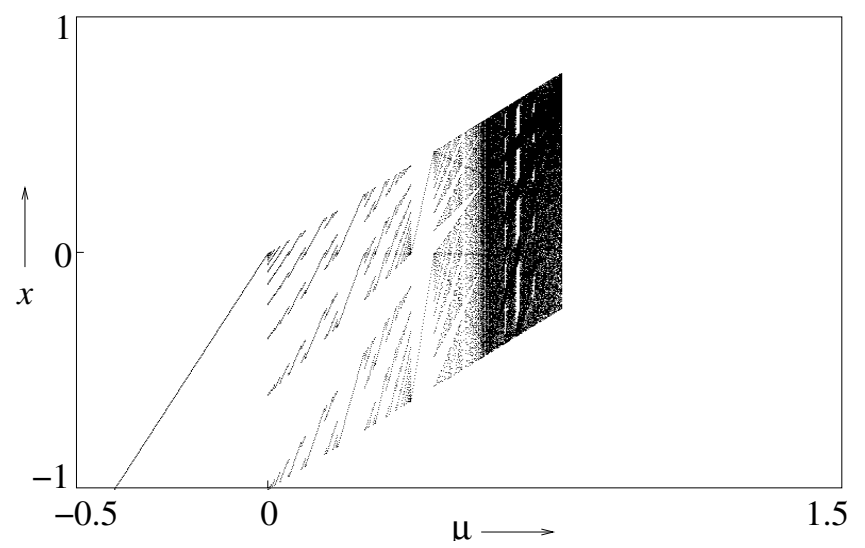

Fig. 3. Bifurcation diagram for the case $0<a<1$ and $b>1$, and $l<0$.

two fixed points exist $-x_{L}^{*}$ unstable and $x_{R}^{*}$ stable - and we get a period-1 orbit with a basin bound$\operatorname{ary}\left(x_{L}^{*}, \infty\right)$.

\subsection{Case 3: $0<a<1$ and

$$
-1<b<0
$$

If the discontinuity is positive, the bifurcation behavior is similar to that in case 1 . There exists stable fixed point $x_{L}^{*}$ for $\mu<-l$ and $x_{R}^{*}$ for $\mu>0$. Both $x_{L}^{*}$ and $x_{R}^{*}$ are stable for $-l<\mu<0 . x_{R}^{*}$ has a basin of attraction $(0,-(\mu+l) / b)$ and all initial conditions outside this domain converge to $x_{L}^{*}$.

An opposite bifurcation behavior is observed for $-1<a<0$ and $0<b<1$ except that for $-l<\mu<0, x_{L}^{*}$ has a basin of attraction $(-\mu / a, 0)$ while all other trajectories converge to $x_{R}^{*}$.

When the discontinuity is negative, the behavior is the same for $\mu<0$ and $\mu>-l$, but for $0<\mu<l$ there is no fixed point. In this range all orbits are bounded but chaos cannot occur because the magnitudes of $a$ and $b$ are less than unity. Since any iterate in $\mathrm{R}$ maps to $\mathrm{L}$, all high-periodic orbits must have only one point in $\mathrm{R}$, i.e. the period3 orbit can only be of LLR type and the period4 orbit can only be of LLLR type, etc. Therefore each high-periodic orbit exists over a single parameter range, and under the condition $0<a<1$ and $-1<b<0$ consecutively higher periodicities have overlapping parameter ranges. This implies that there is a period-adding cascade if $\mu$ is decreased from $-l$ to zero, which is shown in the bifurcation diagram of Fig. 4. The period-2 orbit will exist in the parameter range (3), which extends beyond $-l$, and hence for $\mu>-l$, the period-2

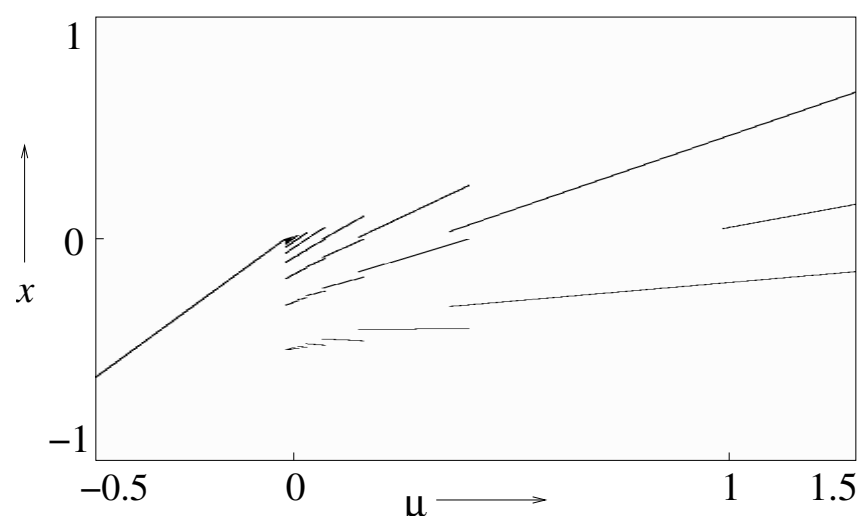

Fig. 4. Bifurcation diagram for the case $0<a<1$ and $-1<b<0$, and $l<0$.

orbit coexists with period-1 orbit, which has basin boundary $(0,-(l+\mu) / b)$.

An opposite bifurcation diagram is obtained for $-1<a<0$ and $0<b<1$.

\subsection{Case 4: $0<a<1$ and $b<-1$}

For positive $l, x_{L}^{*}$ is stable giving a stable period-1 orbit for $\mu<0$. For $\mu>0$, no stable fixed point exists. But since points in $\mathrm{L}$ map to $\mathrm{R}$ and points in $\mathrm{R}$ map to $\mathrm{L}$, orbits remain bounded. There can be high-periodic orbits or chaos depending on the magnitudes of $a$ and $b$ (see Fig. 5).

For negative $l$ the behavior is the same as in Case 3 , except that the period- 1 orbit is not stable for $\mu>-l$. For $0<\mu<-l$, the same period subtracting cascade is observed. However, since the magnitude of $b$ is greater than one, the map becomes stretching and hence chaotic orbits can exist.

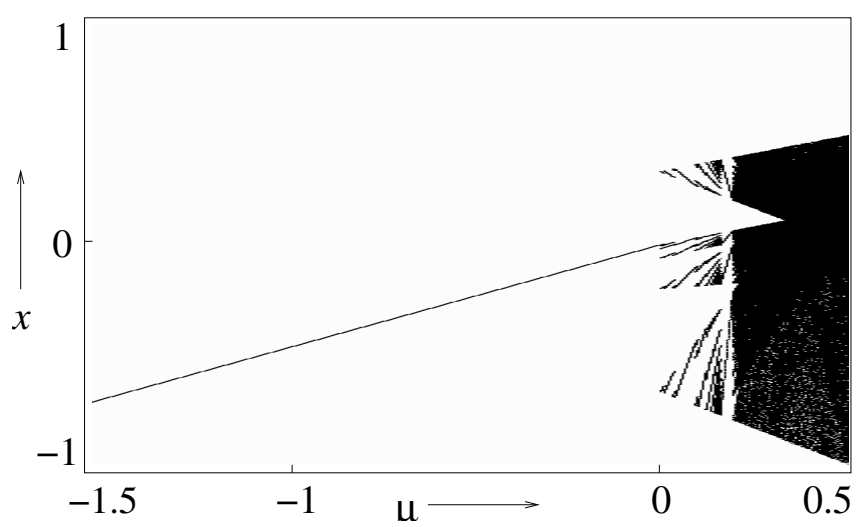

Fig. 5. Bifurcation diagram for the case $0<a<1$ and $b<-1$, and $l>0$. 


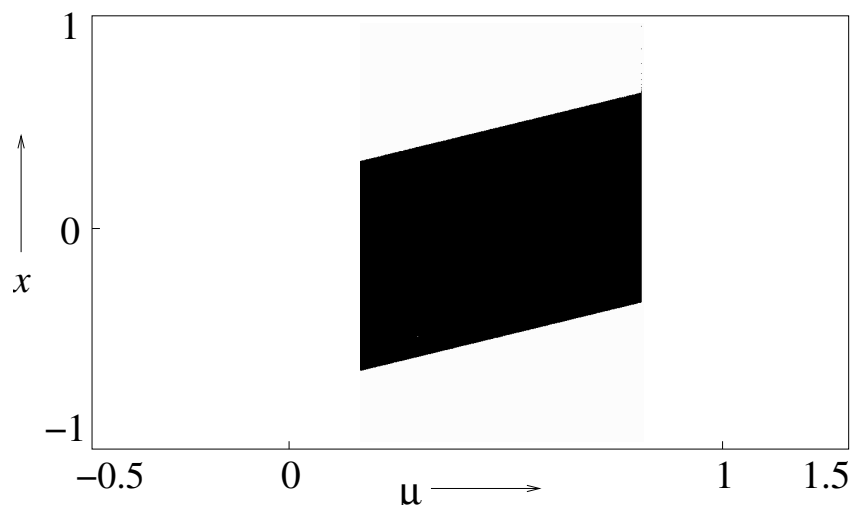

Fig. 6. Bifurcation diagram for the case $0<a<1,0<b<$ 1 , and $l<0$.

An opposite bifurcation behavior is observed for $a<-1$ and $0<b<1$ for both values of $l$.

\subsection{Case 5: $a>1$ and $b>1$}

If the discontinuity is positive, no attractor exists in the entire parameter space. But when the discontinuity is negative, chaos exists for the parameter range $(1-a) l / a<\mu<-l / b$ as can be seen in the bifurcation diagram (Fig. 6). This chaotic attractor has a span of $[\mu+l, \mu]$, with the basin $\left(x_{L}^{*}, x_{R}^{*}\right)$. Initial conditions less than $x_{L}^{*}$ go monotonically towards $-\infty$ while those greater than $x_{R}^{*}$ go monotonically towards $+\infty$. Within the basin boundary, points in $\mathrm{L}$ map to $\mathrm{R}$ and points in $\mathrm{R}$ map to $\mathrm{L}$, and thus the orbits remain bounded. The map however is globally stretching and hence only chaotic orbit can exist. For parameter values outside this range, the chaotic attractor becomes unstable due to boundary crisis. A necessary condition for the existence of chaos is $b<a /(a-1)$; otherwise no attractor exists in the entire parameter space.

\subsection{Case 6: $a>1$ and $-1<b<0$}

For positive $l$, the bifurcation behavior is similar to that for $a>1$ and $0<b<1$ as discussed under Case 2. For $\mu<-l$ no attractor exists and all trajectories go to $-\infty$. For $-l<\mu<0, x_{R}^{*}$ exists and is stable. However in this case it has a basin of attraction $(0,-(l+\mu) / b)$ and all initial conditions outside this range go to $-\infty$. For $\mu>0, x_{L}^{*}$ also exists but is unstable and so the basin of attraction of $x_{R}^{*}$ changes to $\left(x_{L}^{*},\left(x_{L}^{*}-(l+\mu)\right) / b\right)$.

An opposite bifurcation behavior is observed for $-1<a<0$ and $b>1$. The behavior is exactly the same as in Case 2 for $0<a<1$ and $b>1$.
For negative $l$, a stable attractor exists for $\mu>l(1-a) /(a-b+a b)$ (see Fig. 7). This attractor has a basin boundary $\left(x_{L}^{*}, \infty\right)$ beyond which all trajectories go monotonically to $-\infty$. For $\mu$ less than this value, no attractor exists due to boundary crisis and all trajectories go to $-\infty$. Within the above parameter range, high-periodic orbits may exist or the system may be chaotic depending on the values of $a$ and $b$. The period- 2 orbit exists in the parameter range (3), and if $|a b|<1$ this orbit is stable. For $\mu>-l$, a period- 1 orbit is born with a basin boundary $(0,-(l+\mu) / b)$. Figures $7(a)$ and 7 (b) show the bifurcation diagrams for $|a b|<1$ and $|a b|>1$, respectively.

A reverse case exists for $-1<a<0$ and $b>1$ with the difference that the chaotic attractor becomes unstable for $\mu>l(1-a+a b) /(a-b-a b)$ when all trajectories go monotonically to $\infty$. The basin boundary of the chaotic attractor is $\left(-\infty, x_{R}^{*}\right)$. All trajectories outside this basin go to $+\infty$.

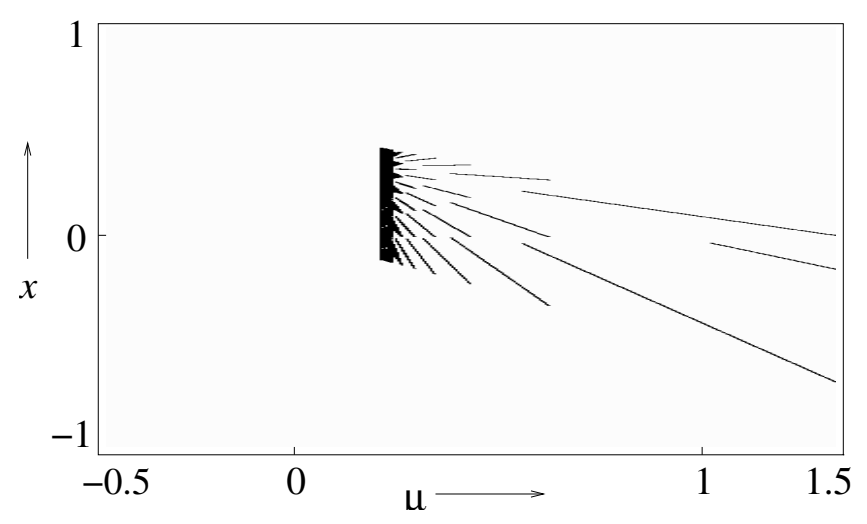

(a)

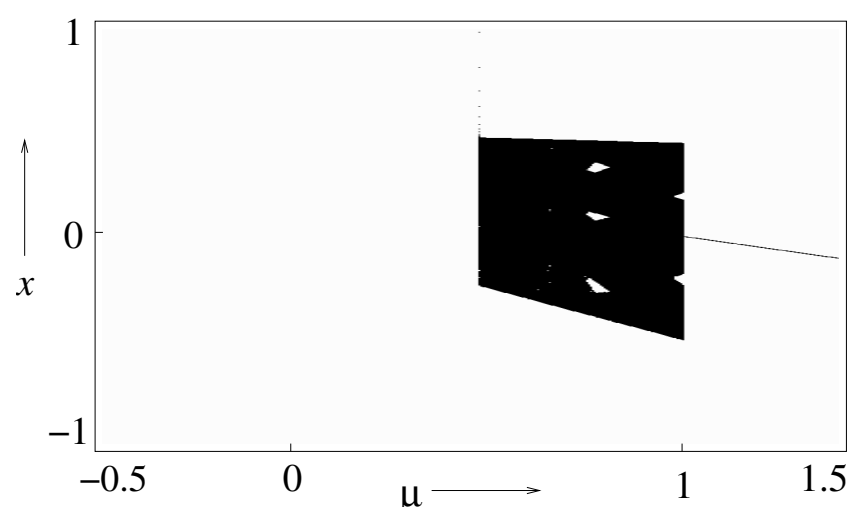

(b)

Fig. 7. Bifurcation diagrams for the case $a>1$ and $-1<$ $b<0$ and for $l<0$. (a) Bifurcation diagram for $a b<1$. (b) Bifurcation diagram for $a b>1$. 


\subsection{Case 7: $a>1$ and $b<-1$}

If the discontinuity is positive, no attractor exists in the parameter range $\mu<0$ and all trajectories go to $-\infty$. However for positive values of $\mu>l(b+1)(1-a) /((b+1)(a-1)+1)$, there exists a trapping region. Since both $a$ and $b$ have magnitudes greater than unity, no periodic orbit can be stable and chaos occurs. The attractor has a basin of attraction $\left(x_{L}^{*}, \mu+l\right)$.

An opposite case exists for $a<-1$ and $b>1$ when no attractor exists for $\mu>-l$ and all trajectories go to $+\infty$. Chaos exists for $\mu<l /((1-b)(a+$ $1)+1)$ with a basin $\left(\mu, x_{R}^{*}\right)$.

When the discontinuity is negative, attractors can exist only when $\mu>l(1-a) /(a-b+a b)$ as in Case 6 . Since no periodic orbit can be stable, the attractor is chaotic (see Fig. 8). This chaotic attractor has a basin $\left(x_{L}^{*}, \infty\right)$ beyond which all trajectories go monotonically to $-\infty$.

A reverse case exists for $a<-1$ and $b>1$ where a stable chaotic orbit exists for $\mu>l(1-$ $a+a b) /(a-b-a b)$ with a basin $\left(-\infty, x_{R}^{*}\right)$, beyond which all trajectories go to $\infty$.

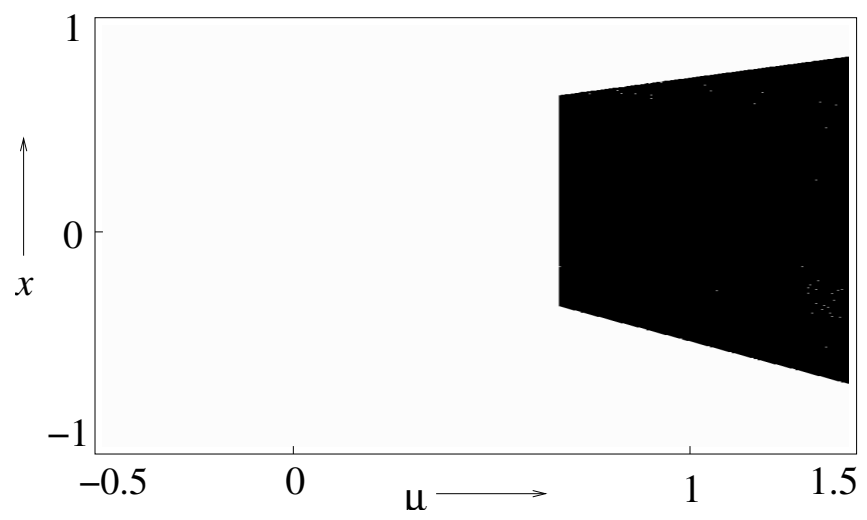

Fig. 8. Bifurcation diagram for the case $a>1$ and $b<-1$ and $l<0$.

\subsection{Case 8: $-1<a<0$ and $-1<b<0$}

For positive values of $l$, the bifurcation behavior is the same as in Case 3, i.e. there exists stable fixed point $x_{L}^{*}$ for $\mu<-l$ and $x_{R}^{*}$ for $\mu>0$. Both $x_{L}^{*}$ and $x_{R}^{*}$ are stable for $-l<\mu<0$ such that $x_{R}^{*}$ has a basin of attraction $(0,-(\mu+l) / b)$ and all initial conditions outside this domain converge to $x_{L}^{*}$.

For negative values of the discontinuity, points in $\mathrm{L}$ go to $\mathrm{R}$ in one iterate and those in $\mathrm{R}$ go to $\mathrm{L}$ in one iterate. Therefore no high-periodic orbit

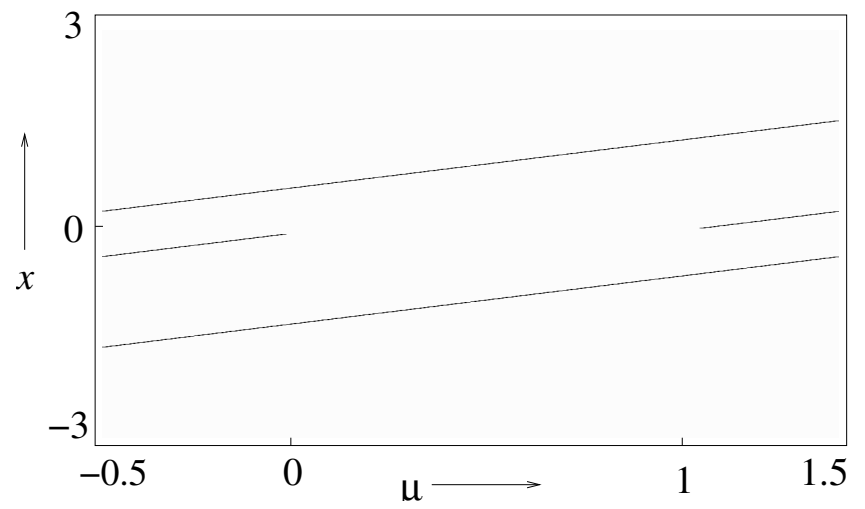

Fig. 9. Bifurcation diagram for the case $-1<a<0$ and $-1<b<0$ and $l<0$.

other than period-2 can exist. The period- 2 orbit is throughout stable since $|a b|<1$. For $\mu<0$ and for $\mu>-l$, there is a coexisting period- 1 orbit as $x_{L}^{*}$ and $x_{R}^{*}$ respectively become stable in these parameter ranges (Fig. 9).

\subsection{Case 9: $-1<a<0$ and $b<-1$}

If $l>0$, the fixed point $x_{L}^{*}$ exists and is stable for $\mu<0$ and thus all trajectories converge to it. For $\mu>0, x_{R}^{*}$ exists but is unstable. Since points in $\mathrm{L}$ map to $\mathrm{R}$ in one iterate and those in $\mathrm{R}$ get mapped to $\mathrm{L}$ after a finite number of iterates, highperiodic orbits can exist. These orbits can have only one point in L, i.e. a period-3 fixed point must be of LRR type which exists in the parameter range (4). The period- $n$ orbit will be stable if $\left|a b^{n-1}\right|<1$. Therefore, if the period- $n$ orbit becomes unstable, no orbit of periodicity higher than $n$ can be stable, and orbit becomes chaotic (see Fig. 10).

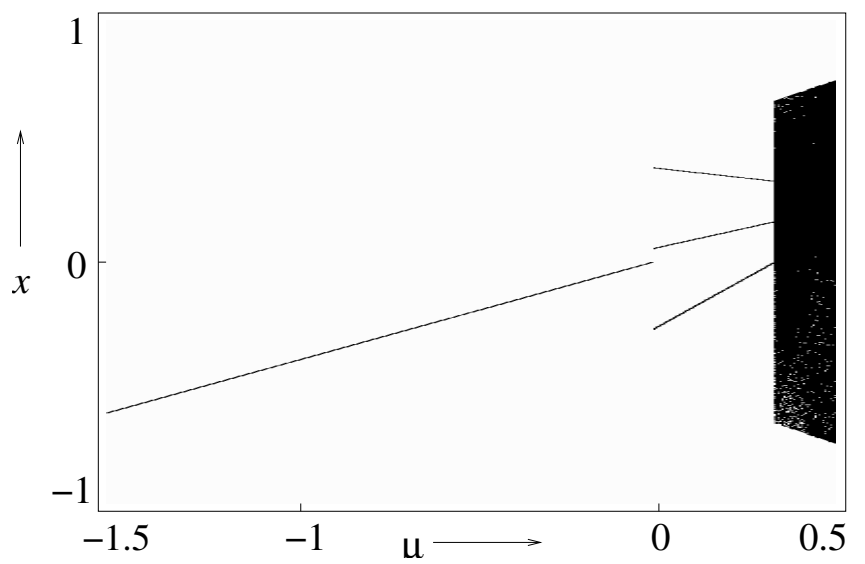

Fig. 10. Bifurcation diagram for the case $-1<a<0$ and $b<-1$ and $l>0$. 
An opposite bifurcation behavior occurs for $a<-1$ and $-1<b<0$.

If $l<0$, the bifurcation behavior is similar to Case 8 except that $x_{R}^{*}$ is unstable for $\mu>-l$. Also the period- 2 orbit is stable in the entire parameter space only if $a b<1$. In case, $a b>1$, no attractor exists for $\mu>0$. An opposite bifurcation behavior occurs for $a<-1$ and $-1<b<0$.

\subsection{Case 10: $a<-1$ and $b<-1$}

Since both $a$ and $b$ have magnitude greater than unity, if an attractor exists it must be chaotic. In case the discontinuity is positive, for $\mu<-l, x_{L}^{*}$ exists while for $\mu>0, x_{R}^{*}$ exists and thus the chaotic attractors are centered around them. For $-l<\mu<$ 0 both the unstable points exist and thus the attractor is evenly distributed. This explains the shape of the chaotic attractors in the bifurcation diagram (Fig. 11). These chaotic attractors have a basin $(((b+1) \mu+l) /(1-a b),((a+1) \mu+a l) /(1-a b))$, and any point outside this range oscillates between $\mathrm{L}$ and $\mathrm{R}$ and goes to $\infty$. The condition for the chaotic attractor to be stable is that

$\mu>\frac{(b+1) \mu+l}{1-a b}$ and $\mu<\frac{(a+1) \mu+a l}{1-a b}-l$

otherwise trajectories very close to the origin map outside the basin boundary and render the attractor unstable.

For negative discontinuity, no attractor exists in the entire parameter space and all trajectories oscillate between $\mathrm{L}$ and $\mathrm{R}$ until it reaches $\infty$.

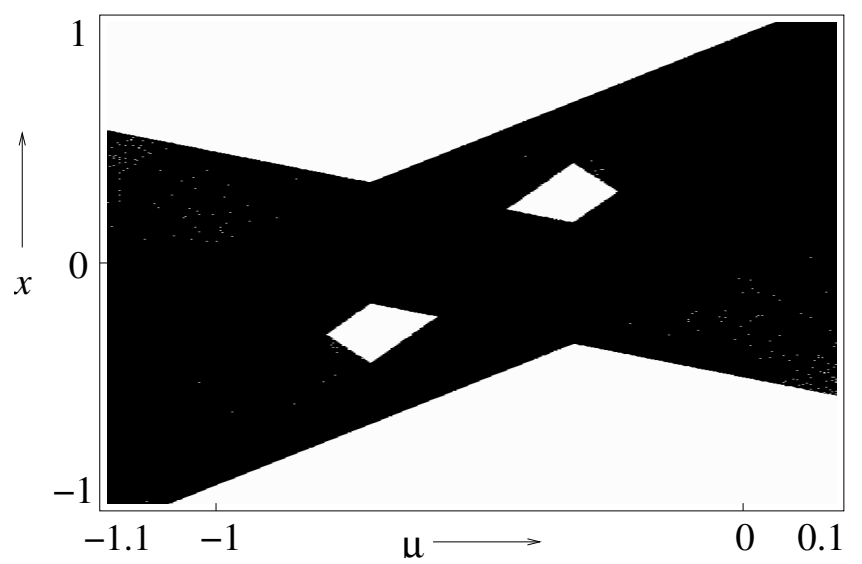

Fig. 11. Bifurcation diagram for the case $a<-1$ and $b<-1$ and $l>0$.

\subsection{Critical values of a and b}

In the above analysis we have excluded the critical parameter values, i.e. where the parameters $a$ or $b$ take the values zero or unity. In such cases the linear approximation does not yield much insight because away from the break-point $x=0$, the slope of the map assumes noncritical values. However, we still need to understand the dynamics of maps with such critical values of the slopes, because there are practical situations which yield piecewise linear maps with such slopes.

The case $a=0$ acts as a boundary between the cases $0<a<1$ and $-1<a<0$ and represents a smooth transition between them. The same is the case when $b$ assumes zero value and $a$ is nonzero. Likewise, the behavior of maps with $a=1$ borders the behavior of maps with $0<a<1$ and $a>1$. For $0<a<1$ and positive $l$, a stable fixed point exists for $\mu<0$. As $a$ approaches unity, the location of the fixed point moves to $-\infty$, and if $a>1$ the fixed point $x_{L}^{*}$ does not exist. However for the particular parameter value of $\mu=0$, the graph of the map coincides with the $45^{\circ}$ line, resulting in the nongeneric situation of an infinite number of fixed points.

Similarly for $a$ or $b$ equal to -1 , the period2 orbit has a slope of unity and so may result in the degenerate case of an infinity of fixed points. These nongeneric situations are however removed by any small perturbation in the map and are not expected to occur in the original system if the accurate discrete-time representation consists of nonlinear segments.

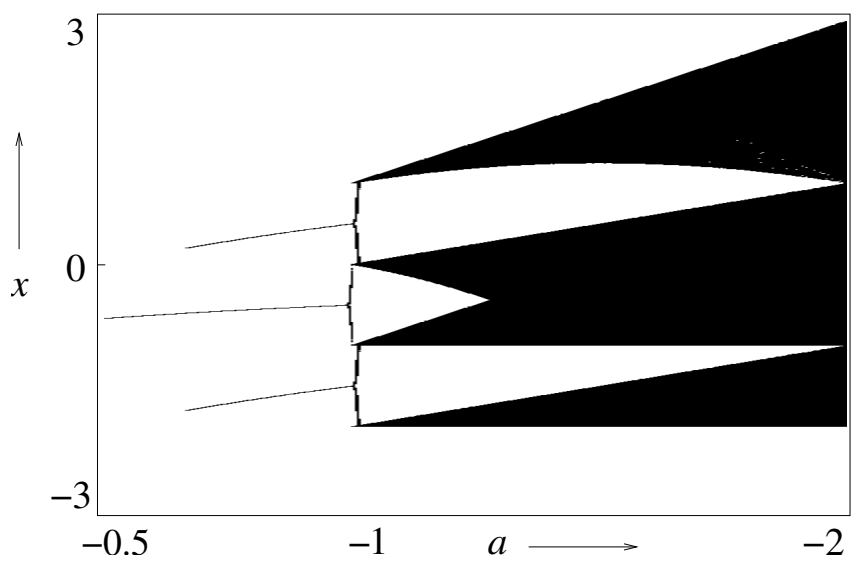

Fig. 12. Bifurcation diagram of the normal form with $b=1$, $l=-1$, and $\mu=1$ held constant and $a$ varied from -0.5 to -2 . 
To illustrate the system behavior for such critical parameter values, we show the bifurcation diagram of the normal form with $b$ set at the critical value of 1 and $a$ varied smoothly through the critical value of -1 (Fig. 12). Note that the nongeneric situation does not arise here as $\mu \neq 0$, and the bifurcation phenomena can be explained with reference to the appropriate cases discussed earlier in this section.

\section{Case Study: Boost Converter with Delay in Control Loop}

As a concrete example of a system that yields a discontinuous map, we consider a well-known power electronic circuit - the current-mode controlled boost converter (Fig. 13). When the switch is on, the current through the inductor builds up linearly till it reaches the specified reference value $I_{\text {ref }}$. The switch opens when $i=I_{\text {ref }}$. When the switch is off, the inductor current falls and the voltage across the inductor reverses in polarity — which now adds to the input voltage to be applied on the load. Once the switch has opened, the next clock pulse causes it to close. Any clock pulse arriving during the on period is ignored. This is the ideal current-mode control logic.

However, the components with which the control logic is realized often have delays and with the industry trend of higher and higher clock frequency, this delay can be significant in comparison with the clock period. Under such conditions, if the clock pulse arrives within a certain delay-period after the switch opening, the state of the switch cannot change and it remains closed till the end of the next clock period. We now proceed to obtain a discrete-time model of this system as observed at every clock instant.

The capacitor helps in smoothening the output voltage, and if the clock period is sufficiently small compared to the $R C$ time constant, the output voltage can be assumed to be essentially constant. Under that condition the system becomes one-dimensional and the inductor current waveform

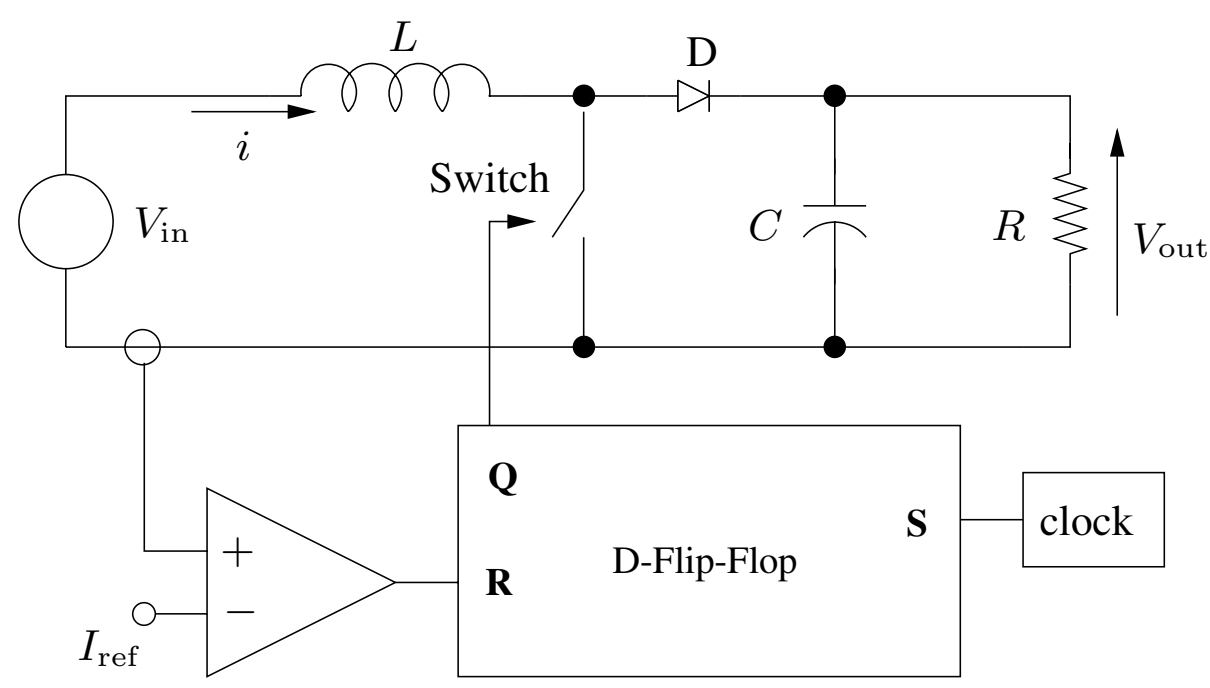

Fig. 13. Schematic circuit diagram of the current mode controlled boost converter.
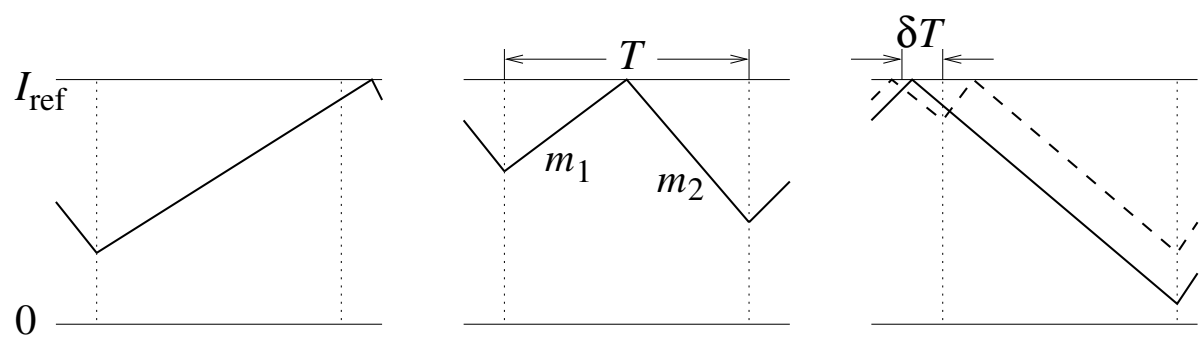

Fig. 14. Possible evolutions between clocks. (a) The switch remains on, (b) Includes on-period and off-period, (c) Solid line: if clock comes before $\delta T$ delay of switch off, and dashed line: if clock comes after $\delta T$ delay of switch off. 
becomes piecewise linear - allowing simplicity in modeling.

Let the slopes of the inductor current during on-period and off-period be $m_{1}$ and $m_{2}$ respectively, and $T$ be the clock period. Let the inductor current at a clock instant be $i_{n}$ and that at the next clock be $i_{n+1}$.
There are two borderlines in the state space (see Fig. 14):

$$
\begin{aligned}
& I_{b 1}=I_{\text {ref }}-m_{1} T \\
& I_{b 2}=I_{\text {ref }}-m_{2} \delta T
\end{aligned}
$$

and the map is obtained as

$$
i_{n+1}= \begin{cases}i_{n}+m_{1} T & \text { for } i_{n} \leq I_{b 1} \\ I_{\text {ref }}\left(1+\frac{m_{2}}{m_{1}}\right)-m_{2} T-\frac{m_{2}}{m_{1}} i_{n} & \text { for } I_{b 1} \leq i_{n}<I_{b 2} \\ i_{n}-m_{2} T & \text { for } i_{n}>I_{b 2}\end{cases}
$$

It is clear that the map is continuous across the border $I_{b 1}$ but is discontinuous across border $I_{b 2}$.

The numerically obtained bifurcation diagram is shown in Fig. 15. The parameter values are $V_{\text {in }}=$ $30 \mathrm{~V}, L=0.1 \mathrm{H}, T=400 \mu \mathrm{s}$ and $I_{\text {ref }}=0.5 \mathrm{~A}$. This gives $m_{1}=300 \mathrm{~A} / \mathrm{s}$, which is kept constant while $m_{2}$ is varied. For $m_{2}<300 \mathrm{~A} / \mathrm{s}$, we have $-1<a<0$ and $b=1$, and negative $l$. Thus we expect a behavior that borders between those of $-1<a<0$ and $b>1$ (reverse of Case 6) and $-1<a<0$ and $b<1$ (reverse of Case 3). In both cases the theory predicts that a stable period- 2 orbit should coexist with the period-1 orbit before the border collision - and that is what we see in the bifurcation diagram of the system.

As $m_{2}$ is increased through $300 \mathrm{~A} / \mathrm{s}$, the slope $a$ becomes greater than unity. So now this is a situation bordering the reverse of Case $7(a<-1$ and

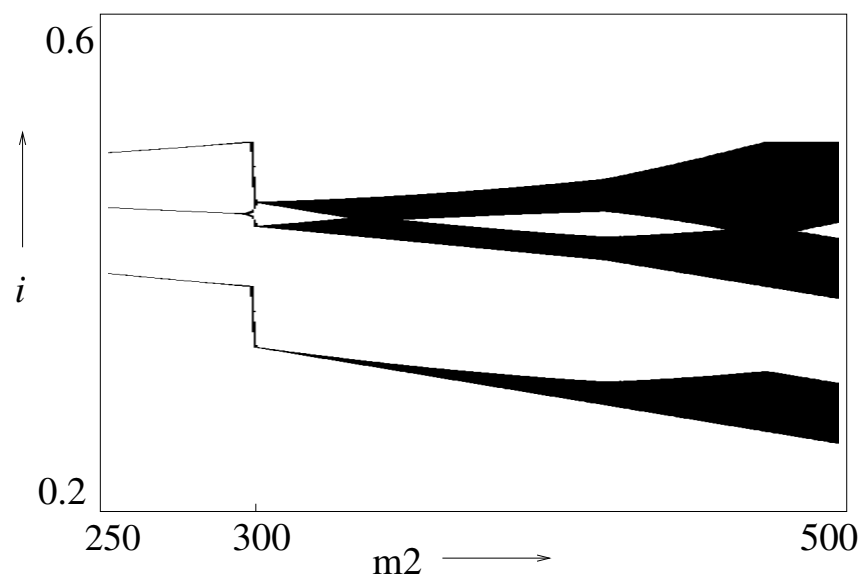

Fig. 15. Bifurcation diagram of the boost converter with delay in control loop. $b>1$ ), with negative $l$. Since all multiples of $a$ and $b$ have magnitude greater than unity, no periodic attractor can be stable and the attractor becomes chaotic. The bifurcation behavior is quite similar to that observed in Fig. 12.

The specific features caused by the discontinuity may be discerned by comparing with Fig. 16, which shows the corresponding bifurcation behavior of the system without delay in the feedback loop.

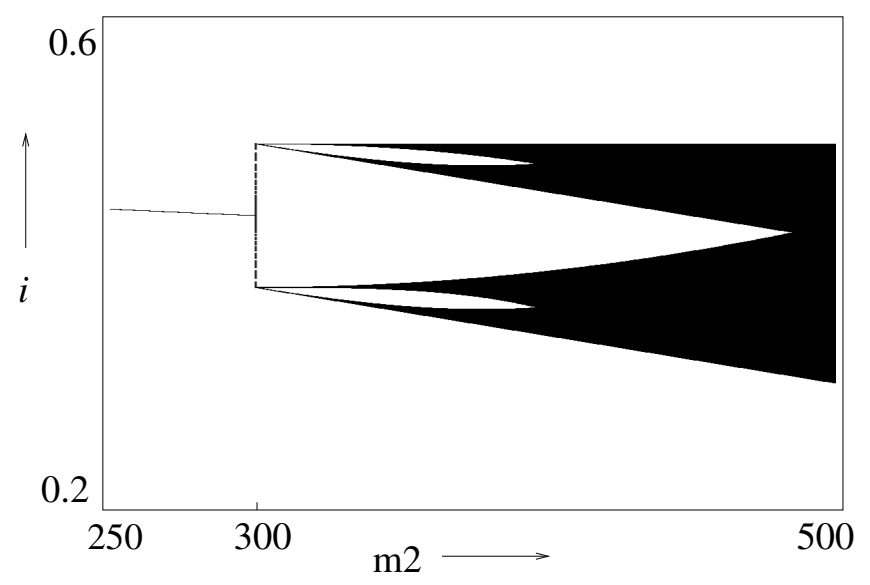

Fig. 16. Bifurcation diagram of the boost converter without any delay in the feedback loop.

\section{Conclusion}

In this paper we have presented the bifurcation behavior of the piecewise linear discontinuous map (2) as an approximation of a general one-dimensional discontinuous map in the vicinity of the point of discontinuity. We have thus developed a classification of the various discontinuous bifurcations depending 
Table 1. Occurrence of attractors in the map (2) as the parameter $\mu$ is varied. The details of the range of existence and stability of the attractors are given in the text.

Asymptotic behavior for positive $l$

\begin{tabular}{llll}
\multicolumn{1}{c}{ Range of $a$ and $b$} & \multicolumn{1}{c}{ For $\mu<-l$} & \multicolumn{1}{c}{ For $-l<\mu<0$} & For $\mu>0$ \\
\hline $0<a<1,0<b<1$ & Period-1 & Coexisting period-1 orbits & Period-1 \\
$0<a<1, b>1$ & Period-1 & Period-1 & No attractor \\
$0<a<1,-1<b<0$ & Period-1 & Coexisting period-1 orbits & Period-1 \\
$0<a<1, b<-1$ & Period-1 & Period-1 & High periodic orbits $/$ chaos \\
$a>1,0<b<1$ & No attractor & Period-1 & Period-1 \\
$a>1, b>1$ & No attractor & No attractor & No attractor \\
$a>1,-1<b<0$ & No attractor & Period-1 & Period-1 \\
$a>1, b<-1$ & No attractor & No attractor & Chaos \\
$-1<a<0,0<b<1$ & Period-1 & Coexisting period-1 orbits & Period-1 \\
$-1<a<0, b>1$ & Period-1 & Period-1 & No attractor \\
$-1<a<0,-1<b<0$ & Period-1 & Coexisting period-1 orbits & Period-1 \\
$-1<a<0, b<-1$ & Period-1 & Period-1 & High periodic orbits/chaos \\
$a<-1,0<b<1$ & High periodic orbits/chaos & Period-1 & Period-1 \\
$a<-1, b>1$ & Chaos & No attractor & No attractor \\
$a<-1,-1<b<0$ & High periodic orbits/chaos & Period-1 & Period-1 \\
$a<-1, b<-1$ & Chaos & Chaos & Chaos \\
\hline
\end{tabular}

Asymptotic behavior for negative $l$

\section{Range of $a$ and $b$}

$0<a<1,0<b<1$

$0<a<1, b>1$

$0<a<1,-1<b<0$

$0<a<1, b<-1$

$a>1,0<b<1$

$a>1, b>1$

$a>1,-1<b<0$

$a>1, b<-1$

$-1<a<0,0<b<1$

$-1<a<0, b>1$

$-1<a<0,-1<b<0$

$-1<a<0, b<-1$

$a<-1,0<b<1$

$a<-1, b>1$

$a<-1,-1<b<0$

$a<-1, b<-1$

For $\mu<0$
Period-1
Period-1
Period-1
Period-1
No attractor
No attractor
No attractor
No attractor
Coexisting periods-1 and 2
Periods-1 and $2 /$ period-1
Coexisting periods-1 and 2
Periods-1 and $2 /$ period-1
Period-2/chaos
Chaos
Period-2/ no attractor
No attractor

For $0<\mu<-l$

High periodic orbits

High periodic orbits/chaos

High periodic orbits

High periodic orbits/chaos

High periodic orbits/chaos

Chaos

High periodic orbits/chaos

Chaos

High periodic orbits

High periodic orbits/chaos

Period-2

Period-2/ no attractor

High periodic orbits/chaos

Chaos

Period-2/ no attractor

No attractor
For $\mu>-l$

Period-1

No attractor

Coexisting periods- 1 and 2

Period-2/chaos

Period-1

No attractor

Periods- 1 and 2/period-1

Chaos

Period-1

No attractor

Coexisting periods- 1 and 2

Period-2/ no attractor

Period-1

No attractor

Periods- 1 and 2/period-1

No attractor on the parameters of the map (2). The results are tabulated in Table 1.

\section{References}

Banerjee, S., Ott, E., Yorke, J. A. \& Yuan, G. H. [1997] "Anomalous bifurcations in dc-dc converters: Borderline collisions in piecewise smooth maps," IEEE Power Electronics Specialists' Conf., pp. 1337-1344.

Banerjee, S., Karthik, M. S., Yuan, G. H. \& Yorke, J. A. [2000a] "Bifurcations in one-dimensional piecewise smooth maps - theory and applications in switching circuits," IEEE Trans. Circuits Syst.-I 47, 389-393.

Banerjee, S., Ranjan, P. \& Grebogi, C. [2000b] "Bifurcations in two-dimensional piecewise smooth maps theory and applications in switching circuits," IEEE Trans. Circuits Syst.-I 47, 633-643.

Banerjee, S. \& Verghese, G. C. (eds.) [2001] Nonlinear Phenomena in Power Electronics: Attractors, Bifurcation, Chaos, and Nonlinear Control (IEEE Press).

Budd, C. \& Dux, F. [1994] "Chattering and related behavior in impacting oscillators," Phil. Trans. Roy. Soc. 347, 365-389. 
Budd, C. [1995] "Grazing in impact oscillators," in Real and Complex Dynamical Systems, eds. Branner, B. \& Hjorth, P. (Kluwer Academic Publishers), pp. 47-64.

Chin, W., Ott, E., Nusse, H. E. \& Grebogi, C. [1995] "Universal behavior of impact oscillators near grazing incidence," Phys. Lett. A201, 197-204.

Di Bernardo, M., Feigin, M. I., Hogan, S. J. \& Homer, M. E. [1999] "Local analysis of $C$-bifurcations in $n$ dimensional piecewise smooth dynamical systems," Chaos Solit. Fract. 10, 1881-1908.

Dobson, I. [1995] "Stability of ideal thyristor and diode switching circuits," IEEE Trans. Circuits Syst.-I 42, 517-529.

Jalali, S., Dobson, I., Lasseter, R. H. \& Venkataraman, G. [1996] "Switching time bifurcations in a thyristor controlled reactor," IEEE Trans. Circuits Syst.-I 43, 209-217.

Maggio, G. M., Di Bernardo, M. \& Kennedy, M. P.
[2000] "Nonsmooth bifurcations in a piecewise linear model of the Colpitts oscillator," IEEE Trans. Circuits Syst.-I 47, p. 1160.

Nusse, H. E. \& Yorke, J. A. [1992] "Border-collision bifurcations including 'period two to period three' for piecewise smooth maps," Physica D57, 39-57.

Nusse, H. E., Ott, E. \& Yorke, J. A. [1994] "Bordercollision bifurcations: An explanation for observed bifurcation phenomena," Phys. Rev. E49, 1073-1076.

Nusse, H. E. \& Yorke, J. A. [1995] "Border-collision bifurcations for piecewise smooth one dimensional maps," Int. J. Bifurcation and Chaos 5, 189-207.

Rajaraman, R., Dobson, I. \& Jalali, S. [1996] "Nonlinear dynamics and switching time bifurcations of a thyristor controlled reactor circuit," IEEE Trans. Circuits Syst.-I 43, 1001-1006.

Yuan, G. H., Banerjee, S., Ott, E. \& Yorke, J. A. [1998] "Border collision bifurcations in the buck converter," IEEE Trans. Circuits Syst.-I 45, 707-716. 